\title{
Design Spaces in Visual Analytics Based on Goals: Analytical Behaviour, Exploratory Investigation, Information Design, \& Perceptual Tasks
}

\author{
Paul Booth \\ University of Southampton \\ pmb1g11@ soton.ac.uk
}

\author{
Nicholas Gibbins \\ University of Southampton \\ nmg@ecs.soton.ac.uk
}

\author{
Spyros Galanis \\ University of Southampton \\ s.galanis@soton.ac.uk
}

\begin{abstract}
This paper considers a number of perspectives on design spaces in visual analytics and proposes a new set of four design spaces, based on user goals. Three of the user goals are derived from the literature and are categorised under the terms exploratory investigation, perceptual tasks, and information design. The fourth goal is categorised as analytical behaviour; a recently defined term referring to the study of decision-making facilitated by visual analytics. This paper contributes to the literature on decision-making in visual analytics with a survey of real-world applications within the analytical behaviour design space and by providing a new perspective on design spaces. Central to our analysis is the introduction of decision concepts and theories from economics into a visual analytics context. Given the recent interest in decision-making we wanted to understand the emerging topic of analytical behaviour as a design space and found it necessary to look at more than just decision-making to make a valuable contribution. The result is an initial framework suitable for use in the analysis or design of analytical behaviour applications.
\end{abstract}

\section{Introduction}

In this paper we propose four design spaces following a review of the visual analytics literature on design spaces and a survey of real-world applications that feature decision-making as a core use case. The proposed design spaces provide a new perspective based on user goals and are categorised under the terms analytical behaviour, exploratory investigation, information design, and perceptual tasks. This paper is part of a larger body of research into analytical behaviour, a topic defined as decision-making facilitated by visual analytics [1]. As we will show, there are many VA applications available today that facilitate decision-making using visual analytics techniques and technology in finance, healthcare, government, retail, and marketing. In the next section, a definition for a design space is introduced, followed by a review of research from visual analytics leading to a new categorisation of the existing literature based on user goals.

\section{Design Spaces}

A design space describes the universe of all possible design choices" [2], constrained by the tools and technologies available as well as the knowledge and experience of the designer. Design spaces have been defined in visualisation and visual analytics (VA) research by de-constructing solutions, revealing design spaces for visualisation types $[3,4]$, tasks $[5,6]$ and the 'visual variables' used for encoding data graphically in maps [7].

The size of the design space (and the number of solutions within it) is expected to be proportional to the complexity of a given problem; an application with multiple dashboards each containing multiple visualisations represents a more complex set of design choices. For any single visualisation there are a number of factors designers must consider that impact the value of the solution. For instance, different bar chart designs impact accuracy [8], the ratio of line-charts and use of dual-line charts effect the choices people make under uncertainty [1] and visual perception can constrain the use of colour in visualisations [9], particularly in maps [10]. In addition, the grouping, quantity, and size of visual marks has been shown to affect 'visual search' perceptual efficiency [11].

Munzner proposes that a design outcome be defined as a solution; the result can be a singular image or a complex application containing multiple interactive visualisations and other non-visualisation components [12]. Munzner also demonstrates that a wider group of starting solutions will increase the number and quality of solutions available to consider, propose, and ultimately select as the optimal outcome. For this reason, by analysing solutions in a design space the 
range of systems, components and processes can be de-constructed to improve the future engineering of solutions in a given context by increasing the number of starting solutions in the space to begin with.

\section{Classifying Design Spaces}

In their research agenda for visual analytics, Thomas and Cook recommended that researchers should "define the design spaces that capture different classes of visualisations" [13, p.72]. Prior to the agenda are a handful of design spaces which classify the basic components and 'language' of visualisation; Bertin's visual variables [7], Cleveland's theory of graphical perception [14], and Mackinlay's method for automated design [15]. These provide the basis for proposals of related interaction patterns [6], data transformations [16], and the systematic classification of visualisation types [17].

Classification of tasks in visualisation and $\mathrm{HCI}$ research has led to the publication of several task taxonomies [6, 18, 19] but we find that tasks are not ideal when defining design spaces. Tasks have been considered as observable low level events such as a mouse click but also relate to sets of semantically-enriched actions such as 'zoom out' or 'delete' [20]. There are significant challenges with inferring high-level goals associated with reasoning and sense-making processes using task analysis, simply because the same low-level tasks are used to achieve a number of different goals. Adding to the challenge; low-level actions tend to be domain agnostic while high-level goals and tasks are domain specific.

Overall, we felt that classifying design spaces by tasks did not align to the way many digital products that feature visual analytics techniques and technologies are designed. This is a critical consideration in order for any proposed spaces to be of use as a tool for the design, analysis or evaluation of real-world applications. Current software product-development processes in start-ups and large organisations often apply user-centric methods such as Design Thinking to identify and validate products and features that focus on the goals or 'jobs' of users.

In their classification of tasks within the visualisation design space, Schulz [5] proposes a set of five dimensions; WHY (the goal), WHAT (data characteristics), WHERE (in the data does the task operate), WHO (is the type of user), and WHEN (is the task performed/the order of tasks). Their view of goals is further divided into three levels; exploratory analysis (e.g. undirected search), confirmatory analysis (e.g. directed search), and presentation. In our review of the broader literature, we are not aware of any research that classifies decision-making as a goal. In the next section, we take Schulz's view of goals and combine it with three common goals from identified in the literature and propose analytical behaviour as a fourth goal.

\section{Classifying Design Spaces by User Goals}

In this section we combine the user goals from Schulz [5], Thomas and Cook's VA agenda [21], and Munzner [12]. A summary table is provided in Table 1, showing three goals grouped into perceptual tasks, exploratory investigation, and information design.

Thomas and Cook assert that the aim of visual analytics is the production of insights, enabling people to 'detect the expected and discover the unexpected', produce informed assessments, and the communication of results to a range of audiences [21, p.10]. Muzner [12] proposes three goals that map Thomas and Cook's agenda; discovery (exploring unknown data), confirmation (hypotheses using partially known data) and presentation/communication (methods for sharing information with others). Schulz et al. [5] provide three types of goals in the visualisation design space; exploratory analysis (undirected search), confirmatory analysis (e.g. directed search), and presentation. [5].

A summary table is provided in Table 1, grouping classifications together under three goals we use in the remainder of this paper, with the addition of a fourth goal related to decision-making not previously considered in the literature.

The following sections discuss the goals, agents, methods and functionality, and also the technology and techniques present in the above three design spaces. This approach aligns to the goals, the 'what' and 'who' applied by Schulz, while further expanding on the importance of considering the technology and techniques available in each space. The following lists the five dimensions that we find are common to all four design spaces proposed in this paper. These dimensions are also applied in the survey of real-world applications relating to decision-making in the next section. The

\begin{tabular}{|c|c|c|c|}
\multicolumn{2}{c|}{ Table 1. Table of User Goals } \\
\hline Goal & $\begin{array}{c}\text { Thomas and } \\
\text { Cook } \\
(2005)\end{array}$ & $\begin{array}{c}\text { Shulz et al. } \\
(2013)\end{array}$ & $\begin{array}{c}\text { Munzner } \\
(2015)\end{array}$ \\
\hline $\begin{array}{c}\text { Perceptual } \\
\text { task }\end{array}$ & $\begin{array}{c}\text { Detect the } \\
\text { expected }\end{array}$ & $\begin{array}{c}\text { Directed } \\
\text { Search }\end{array}$ & Confirmation \\
\hline $\begin{array}{c}\text { Exploratory } \\
\text { investigation }\end{array}$ & $\begin{array}{c}\text { Discover the } \\
\text { unexpected }\end{array}$ & $\begin{array}{c}\text { Undirected } \\
\text { Search }\end{array}$ & Discovery \\
\hline $\begin{array}{c}\text { Information } \\
\text { Design }\end{array}$ & Communicate & Presenation & $\begin{array}{c}\text { Presentation \& } \\
\text { Communication }\end{array}$ \\
\hline
\end{tabular}


purpose of this dimensional framework is to support a structured analysis and comparison of design spaces and not to empirically classify the proposed spaces.

Agents: Whether human or machine, with consideration of features of the agent and their relationships including; division of labour, roles, autonomy, intelligence, and initiative

Methods/Functionality: What the agent is able to do, limited by external constraints (technology and techniques) and internal cognitive constraints (knowledge, preferences, role, reference-point)

Outputs: The goal as an observable artefact - actions, choices, decisions, behaviour, or knowledge artefacts

Technology: Web, mobile, cloud platform services, paper, user interfaces (touch screen, voice, gestural, physical)

Techniques: visual encoding, interaction, narrative, user experience design, user interface design

There are two agent types we are concerned with the human and computer. Their specific roles, autonomy and relationships may vary across solutions both across or within design spaces. Behaviour and functionality for solutions may be limited by a range of constraints such as the value and type of output. The specific technologies and techniques used in analytic processes of all four design spaces will vary according to the type of device (i.e. mobile, desktop) which will limit the inputs available (i.e. touch, mouse, voice). Our analysis of three design spaces in the next section reveals the design spaces to be well-defined with considerable variation between solutions within the same design space. This is also the case in the later survey of real-world applications in the analytical behaviour design space.

\subsection{Exploratory Investigation}

In the design space of exploratory investigation, the goal is a process of discovery that leads to insight. A valuable outcome could be either knowledge or evidence to support taking action or to support continued exploration. Examples of this design space relate to work in intelligence analysis [13, 22] and finance [22].

The agents in exploratory investigations are the analyst and the computer where the computer includes the platform, device and applications. Dividing entities this way is common in previous research and real-world scenarios [23, 24, 25]. The outputs of exploratory investigations are knowledge, insights, and information $[26,27]$.
The technology in this space applies to both structured and unstructured data [21]. During reasoning and sense-making processes, tools exist to support the generation meta-data in the form of field notes, hypotheses, or tags to classify information and save it in the application. Techniques used in intelligence analysis and fraud include the use of network visualisations that enable analysts to examine the relationships among data points [28, 29]. Other techniques structure solutions around Pirolli and Card's sense-making model [30], combining statistical modelling with sense-making under the proposed 'semantic interaction design space' [31] and mixed-initiative and recommender techniques $[32,33,34]$.

\subsection{Perceptual Tasks}

In perceptual tasks, the goal is to efficiently and effectively support the accurate performance of decoding values from visualisations. The term 'task' in visualisation and visual analytics research has a broad meaning that extends beyond perception; tasks have been considered a design space of their own in previous research attempting to unite multiple task taxonomies [5]. Unification and systematisation are important aims of task research, given its broad scope as a topic that includes semantic classification [20], interaction techniques [6], and the classification of patterns of user actions $[35,36]$.

Perceptual task-based analysis features the same agents as exploratory investigation: a user and a computer (which includes its applications). Task taxonomies have been applied to track the activities of users in the perceptual task design space, leading to systems that offer visualisation recommendations [34] and mixed-initiative tools that observe and adapt to the task the user is performing [32]. These examples also appeared as solutions in the exploratory analysis design space. Both provide a means to optimise the effectiveness of perceptual and analytic tasks, while also providing an overall tool for users to 'detect the unexpected'. In this regard, we find that perceptual-task solutions may also exist as solutions in other design spaces.

There is a distinct class of output noticed with perceptual tasks that we refer to as observable objective action - such as mouse clicks and other behaviour that are objectively measurable. There are many examples of objective actions such as when a user is identifying changes in stock price using tree-maps [35], or finding the cheapest flights [37], finding travel accommodation to fit constraints [35], evaluating medical data [38], making military logistics assessments [24] or evaluating 
weather alert severity [39].

In all the above examples, the task is to perceive an objective value accurately rather than make a subjective economic choice or judgement. For example, it is objectively true as to which flight is the cheapest, or the severity of the weather, or identifying certain logistical requirements. However, What follows a perceptual task may be a decision that takes into account additional economic context, such as whether the cheapest flight available has a longer travel time, or that the risk of a high return stock is less favourable to a conservative investor than a low-risk stock with lower expected returns. These examples highlight the gap in the literature around decision-making which is addressed by new research into analytical behaviour [1].

\subsection{Information Design}

The information-design space is exemplified by data journalism and enlightening reports that present a compelling answer to a question, underscoring Bertin's view that information is a reply to a question [7, p.11]. Information design solutions demand no action or decision-making from their 'readers'. Instead, an often controlled narrative is presented in a visually engaging way. A design space for narrative visualisation has been proposed previously with three features: a taxonomy of genres, visual devices that facilitate the narrative, and narrative structure [4]. As noted in these and other real-world examples, aesthetic choices are a dominant component of a solution, used to increase engagement with the content by making it more visually compelling than statistical charts and graphs.

Information design solutions - often referred to as info-graphics - are commonly used in print and web-based journalism [4], by intelligence analysis using geographic data [40], and in early print visualisations such as Snow's Cholera map and Minard's depiction of Napolean's Russian campaign [41]. A more complete range of info-graphics that fit the information design space can be also seen in mainstream monographs [42, 43, 44, 45, 46, 47].

Narrative techniques are used extensively in info-graphics [4, 48, 49], but have also been applied to aid the sense-making process in intelligence analysis [29]. However, narrative can become rhetoric, making info-graphic solutions prone to framing effects $[50,51]$. Intentional or not, the reader can be mislead or misinformed when certain techniques are applied [52].

In print format an info-graphic has only one agent; the human, who engages but does not interact physically with the image. For info-graphics on the web, there are two agents to consider the user and the computer/application. Unlike in the exploratory investigation design space, information design solutions are used mostly to inform the audience and sometimes offer users a limited exploratory experience due to the constraints imposed by having an opinionated design and the need to control the underlying narrative.

\subsection{Analytical Behaviour}

Analytical behaviour is defined as decision-making facilitated by visual analytics [1]. Decision science covers a broad set of topics and fields, but analytical behaviour is (presently) constrained to an economic perspective of choice and judgement. The analytical behaviour design space includes the decisions people make that occur after a task or as the result of an exploratory investigation. For example, identifying the current price of a stock or a change in price are strictly perceptual tasks, but choosing which stock to invest in or judging how much it may be worth in the future is a purely economic decision facilitated by visual analytics techniques and technology. In the next section we explore and define analytical behaviour by surveying real-world applications that facilitate decision-making.

\section{Analytical Behaviour Design Space}

In this section we survey several real-world applications to examine and define the analytical behaviour design space. As with the three design spaces outlined in the previous section, we consider analytical behaviour in the context of the same five dimensions; agents, methods/functionality, outputs, technology, and techniques. Since the fourth design space analytical behaviour does not exist in the literature, it was determined that a survey of real-world applications for decision-making was the most appropriate method for de-constructing this unique design space.

Economic decision making is a vital part of analytical work. Real-world examples of analytical behaviour explored in the next section of this paper include financial investing, online auctions, personal finance, travel, and healthcare. Focusing on economic topics related to decision-making in a visual analytics context, this section encourages new research into analytical behaviour as a design space.

\subsection{Survey}

In the following sections, several real-world applications are presented as examples of the analytical behaviour design space. The assertion is that these products facilitate decision-making with visual analytics techniques and technology. We explore multiple 
contexts of analytical behaviour including investing, online auctions, personal finance, and travel.

The survey introduces economic concepts that are relevant to decision-making in the context of visual analytics. The five dimensions used to evaluate the previous three spaces cannot be applied as easily, due to the lack of literature available compared to the previous three design spaces. In the following survey of analytical behaviour applications, we still consider the agents, but must infer some functionalities, technologies, techniques and outputs given the limited time and documentation available for these applications. An overview of each application, a description of how data is presented and an outline of relevant economic concepts is provided to contextualise analytical behaviour.

\subsection{Investment Applications}

Investing money in the stock market is an obvious example of economic decision-making. There are numerous applications targeted at both professional and amateur investors on a variety of platforms. The two applications examined here are both mobile applications available on mobile platforms and aimed at amateur investors.

Acorns is a micro-investing mobile application launched in the US in 2015. The app connects to a person's bank account and "rounds up" the change from everyday purchases to use as investment capital. Users have three levels of risk to choose between ranging from 'conservative' (low risk/lower expected return) to 'aggressive' (high-risk/higher expected return), shown in Figure 1.

RobinHood is an alternative trading application that offers free stock trading in the US and Australia (currently), real-time market data, and the ability to execute trades quickly. Data is presented using line charts, and spark-lines (shown in Figure 2). The app also offers notifications on earnings and dividends.

Both applications are examples of analytical behaviour given that the user is encouraged to make investment decisions based on data visualisations and other information.

Economics research has shown that people are sensitive to changes in value, namely gains and losses relative to a neutral reference point Kahneman:2002tb, Kahneman:1979wl. The two trading apps represent gains and losses in different ways. RobinHood uses colour to encode both chart-lines and text as gains (green) and losses (red). The Acorns app provides an overall "total" for the user's account as a gain or loss. Line graphs are used in both apps to show prices over

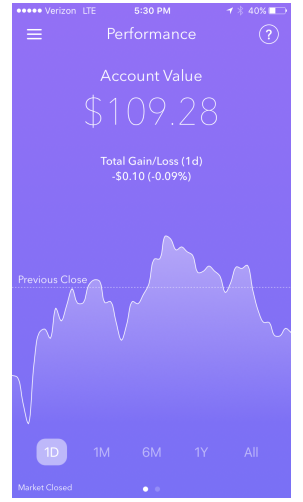

Figure 1. Acrons mobile app trading platform

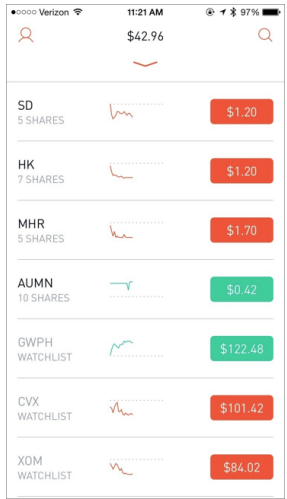

Figure 2. RobinHood mobile app trading platform time though neither app includes a visible $y$-axis in the main charts. Both have the option to change the time range between one day, aggregated monthly periods, and a yearly view.

Reference points can be set by future expectations [53] meaning that an expected future gain that is never received can evoke a similar feeling to losing something they own. The "moderate" investment strategy option in the Acorns app sets the users expectation of capital return over a period of years, this may be too distant to be considered an expectation reference point but not as a goal - a reference point that could lead to greater disappointment given the time taken to reach long-term investing goals.

\subsection{Online Auctions}

Terapeak, shown in Figure 3, uses data from eBay to provide analytics-as-a-service for sellers to help them decide what to sell, in what quantities, and at what price. Terapeak gives insights about return and risk information at the product level on the rate of sales, pricing, competitor rankings, and demand trends - all relevant economic topics. By using Terapeak, users have access to more market pricing information which is likely to affect there choices and judgements when trading goods.

The endowment effect is a concept from economics that states that sellers will set higher-than-market prices because ownership leads to over-valuation [54, 55]. Access to data about the market may reduce the endowment effect by reducing the user's "bounded rationality" [56, 57]. Without market data, buyers and sellers have to make highly subjective, biased choices - thereby increasing the potential for people to fail to recognise the optimum decision strategy compared to 


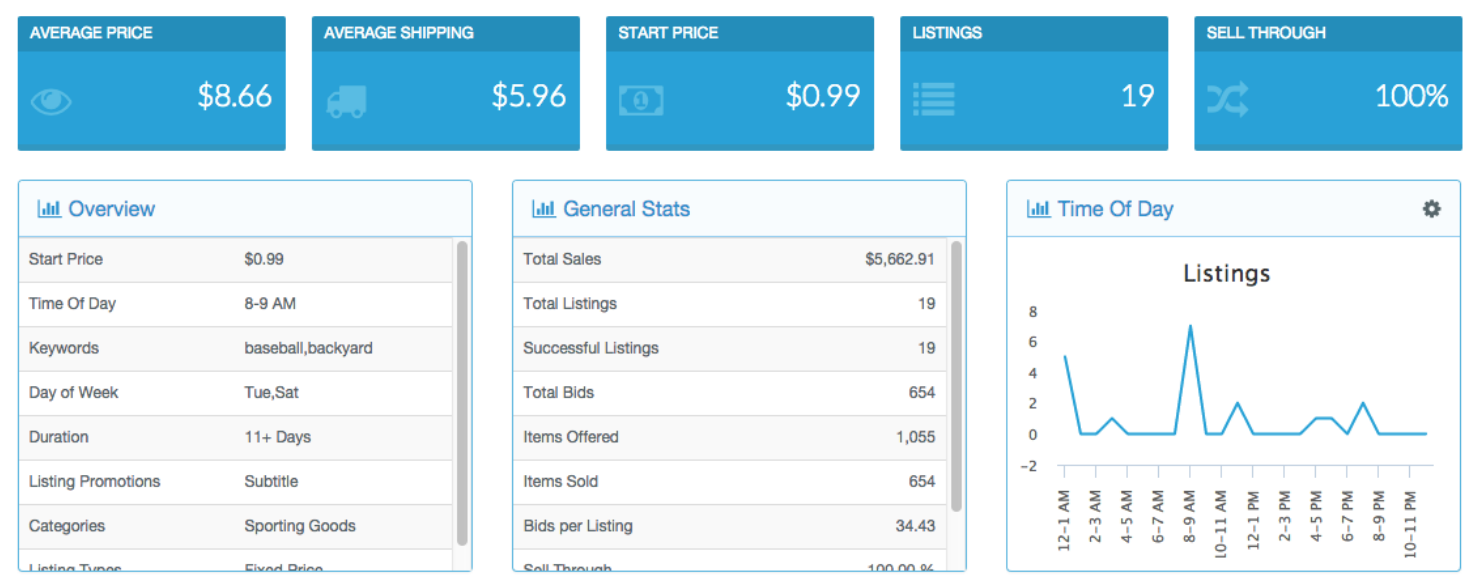

Figure 3. Terapeak: Analytics-as-a-service for eBay auctions to help buyers and sellers make trading decisions

those with access to more information.

However, when people are aware of the average price an item sells for, they may feel the effect of the winner's curse if they have overpaid for the item [58]. In the case of the loser's curse, a bid below the winning price or the price paid is inflated above the value of the product, resulting in low (or negative) profits. The use of visual analytics for portfolio selection has been shown to improve profit maximising decisions [58], which would seem to relate directly to the analytical behaviour that Terepeak facilitates.

\subsection{Banking}

The day to day management of our personal finances has evolved in the light of internet and mobile technologies, alongside other factors that
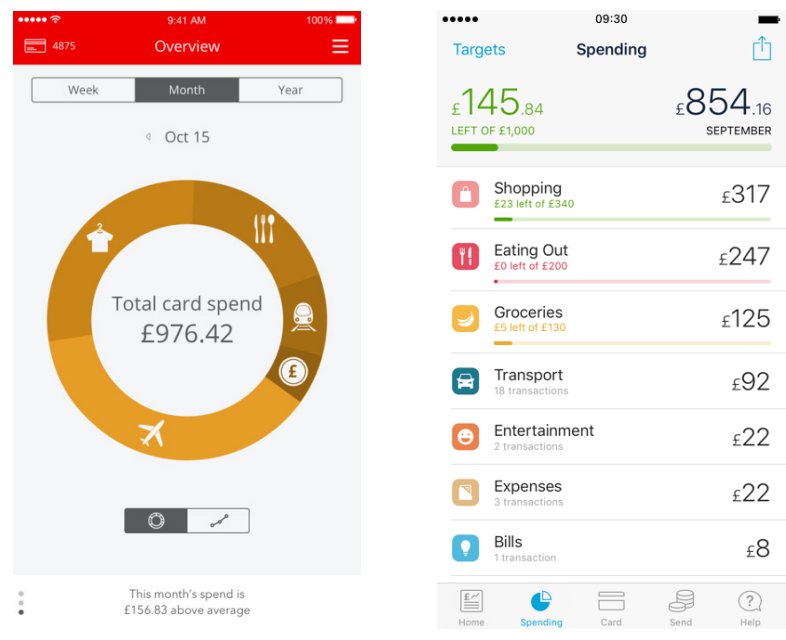

Figure 4. The Spendlytics (left) and Monzo (right) mobile banking apps drive innovation in competitive markets. In the following section we survey two personal finance mobile applications from Santander and Monzo.

Gains and losses, as we have already mentioned, are perceived relative to a reference point. Time is a reference point in both apps, but Monzo also uses budgets and goals that act as reference points for spending and saving money.

Goals are displayed as text and a single bar line across the screen. This visualisation technique supports perceptual tasks such as identifying and comparing values. Users can set custom budgets for 'groceries' and 'leisure' for example, but in each case, the bar used to express the total always spans the width of the device screen. In doing so, a person can quickly identify spend as a proportion of the screen width. The number shown beside each budget line shows the absolute amount spent.

Beside the budget value for each bar in Monzo is the term "left" (i.e. "you have 50 remaining"), which frames the user's spending. This is an example of "attribute framing" [59], for which there is some preliminary research in visual analytics [51, 60]. As a user's spending in Mint increases the coloured bar increases in size starting from the left of the screen, focusing on the cumulative spend. However, as spending in Monzo increases the thin bar reduces in size from right to left, visualising what is left in the budget. This technique highlights an opportunity for further research into the visual encoding of framing for analytical behaviour.

\subsection{Travel}

In this section, we look at analytical behaviour present in the flight search tool SkyScanner and the accommodation platform Airbnb, in relation to a 


\begin{tabular}{l|cccccccc|ccccc}
\multicolumn{1}{c}{} & Table 2. Table of Constraint Sets for Travellers \\
Set & A & B & C & D & E & F & G & H & I & J & K & L & M \\
\hline Cost & 1 & 0 & 0 & 1 & 1 & 0 & 1 & 0 & & & & & \\
Time & 0 & 1 & 0 & 1 & 0 & 1 & 1 & 0 & X & X & X & 0 & 1 \\
Location & 0 & 0 & 1 & 0 & 1 & 1 & 1 & 0 & 0 & 1 & X & X & X
\end{tabular}

collection of constraint sets devised from our own analysis.

Traditional economic thinking embraces the rational choice of individuals, meaning that people are expected to choose between alternatives in line with well-defined preferences. When searching for a holiday, the user must consider certain constraints that map to their own preferences; the cost (i.e. cabin class and airline), time (i.e. departure and arrival dates), and location (i.e. airport).

We examined around a dozen travel sites and considered the possible constraint permutations that a user might have when they begin to use a travel service. Table 2 shows eight permutations of constraints sets (columns A-H) where $1=$ true for a given constraint and $0=$ false. Six additional constraint sets (I-M) assume a fixed budget (shown as blank in the cost row) and provide a set of 'fuzzy' constraints ( $\mathrm{X}=$ fuzzy) relating to time and location, meaning the user may provide a range of dates instead of specific dates for outbound and return flights, or a range of locations by choosing a region, country, or continent instead of a single airport. Both SkyScanner and Airbnb sites provide an interface for preference sets $F$ and $G$ as part of a standard search interface. Skyscanner has designed its interface for sets F, G, and I-M, but Airbnb is limited to only F, G, L and $M$ because the user must provide specific travel dates which is optional on SkyScanner.

Airbnb and SkyScanner both use additional VA

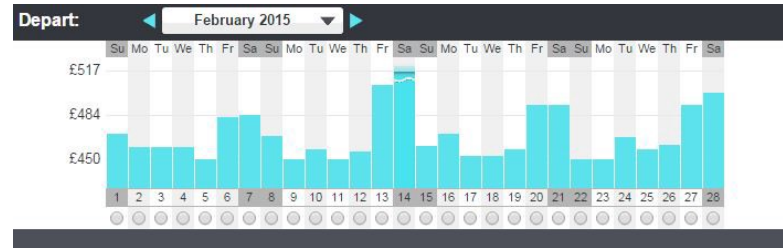

Figure 5. A visualisation used on the SkyScanner website to support users to choose flight dates. The height of each bar is the price of a flight that day.

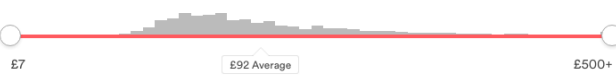

Figure 6. An interactive histogram of rental prices used on the AirBnB website techniques to provide users with choices based on their constraints. In the Skyscanner chart shown in Figure 5, either the lowest or highest price could be taken as a reference point by the user. A flight of average cost could potentially feel like a gain or a loss depending on the users reference point. By comparison, AirBnBs (current web) interface features an interactive histogram (Figure 6) with three potential reference points; the lowest, highest, and average price. These are just two examples of representing and framing pricing information, highlighting the importance to better understanding how visualisations inform analytical behaviour.

Framing effects are a concept familiar to both economics [59, 61] and visual analytics [50, 62, 51]. Framing effects occur when the same data presented in different ways alters a person's perception of the data. Attribute framing uses an attribute of an object or event as the focus of a framing manipulation such as with ratings (e.g. good-bad), outcomes (e.g. success/failure), and product quality (e.g. lean/fat content in meat).

Attribute framing is used by three travel websites to frame the "availability" of listings. Booking.com (Figure 7) and Hotels.com (Figure 8) use what Levin [59] refers to as a 'positive frame' for availability; the amount booked or reserved. Airbnb uses the 'negative frame' to indicate how many are left (Figure 9).

Both frames can be used to generate a sense of urgency in the user by highlighting scarcity in the market. Although in the case of Hotels.com the data is used more informatively since even low numbers

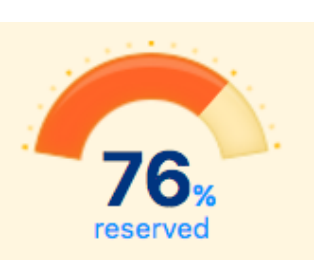

Figure 7. Booking.com

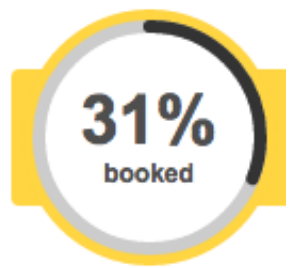

Figure 8. Hotels.com
Only $11 \%$ of listings are left for these dates. We recommend booking a place soon.

Figure 9. AirBnB.com 
are shown in the same way. Though we were unable to establish the exact limit, the frame changes above $60 \%$ for booking.com and at or below 25\% for AirBnB. When availability is not low, both Booking.com and Airbnb remove the visualisations, choosing to focus attention on the volume of properties available, giving a the user a sense that they have a wide range of choices.

\section{Summary}

This paper has considered a new perspective on design spaces in visual analytics and proposed a new set of four design spaces based on user goals.

There already exists a body of research on design spaces in visualisation and visual analytics that includes a definition of spaces, solution scope, and a number of approaches to their classification. The initial motivation was to understand and explore the design space for analytical behaviour, defined as decision-making facilitated by visual analytics. However, we determined the value of characterising other spaces in the same way was greater and looked to expand the work accordingly by considering spaces to be related to user goals.

Looking at the original visual analytics agenda and other publications from the field, we considered the goals and aims of visual analytics proposed by other researchers. Three common goals were established as a result; perceptual tasks, exploratory investigation, and information design. For each of these three goals we review the literature, not as an empirical classification but in terms of a dimensional framework to structure the discussion. Each space was considered in terms of its agents, method/functionality, outputs, as well as the technology used and the techniques that underpin interactions, narrative, user experience and visual encoding.

Decision-making is somewhat overlooked topic for study within visual analytics. As a contribution to the study of analytical behaviour (decision-making facilitated by visual analytics), this paper surveyed a set of real-world applications and presents a discussion. The survey highlights the need for further empirical research into analytical behaviour and evaluation methods for analytical behaviour solutions.

There are any number of ways to classify design spaces, user goals being just one. Peoples goals as we have defined them here are likely change over in any session. It is possible for the same solution to appear in two design spaces and for multiple spaces to be used in one application. During analysis, a user's goal (as we have defined them) can move from a perceptual task to exploratory investigation. In fact, it may be more accurate to say that perceptual task often form the basis of other tasks. Perceptual tasks occur when users are engaged with information designs or to support decision-making. Essentially, our approach acknowledges that Visualisations and visual analytics tools can be used in multiple ways to achieve multiple goals and that some goals occur before (or within) others. It makes sense then, that whole or partial solutions from one design space would appear in another if they are useful to achieve multiple goals. For example, the same visualisation may serve as both information design and as a tool to facilitate decision-making.

In our own research into analytical behaviour, we have found both the process and outcome of this work to be valuable. We have not provided, or set out to provide, an empirical classification or a systematic taxonomy. However, the immediate value to visual analytics researchers is the contextualisation of decision-making into a design space. By reviewing a limited set of real-world applications we hope that other researchers see there is no shortage of examples of decision-making is being facilitated by visual analytics techniques and technology and begin to extend this as a research topic.

Part of what makes this analysis unique is that it focuses on the value of the outcome or the user's perception of value, whereas visual analytics research has typically targeted the actions of users. Task analysis, for instance, is concerned with classifying types of actions, not the economics of choices and judgements made under uncertainty (or otherwise). We suggest that the real-world examples presented in this paper show the importance of considering how decisions are informed by the perception of gains and losses relative to a reference point. Framing effects have also been shown to provide insights into the way travel sites promote scarcity to influence purchases.

There are certainly questions that need addressing in future research around representation effects. There are no studies we are aware of on the most effective framing method for market scarcity, for instance. Although there are many ways to record user experience data (e.g. clicks, and page views), there is a potentially large volume of data available to study and optimise visualisations for analytical behaviour in real-world applications.

In conclusion, there are many decision-making problems to be solved and better understood that are already being used in popular mobile and desktop applications. We have shown that these solutions come from a design space which utilises visual analytics technologies and techniques. However, we also emphasise that these solutions are best evaluated using a decision-theoretic perspective, such as economics. 
However, not all problems and solutions we reviewed (but ultimately did not include in this paper) qualified as 'analytical behaviour'. The divisive factor for classifying analytical behaviour became whether the application captured the decision of the user as well as informing the user's decision with interactive data visualisations.

\section{References}

[1] P. Booth, N. Gibbins, and S. Galanis, "Representation Effects and Loss Aversion in Analytical Behaviour: An Experimental Study into Decision Making Facilitated by Visual Analytics," in The Hawaii International Conference on System Sciences, Hawaii International Conference on System Sciences, Jan. 2018.

[2] H. Schulz, S. Hadlak, and H. Schumann, "The Design Space of Implicit Hierarchy Visualization: A Survey," IEEE Transactions on Visualization and Computer Graphics, vol. 17, no. 4, pp. 393-411, 2011.

[3] S. K. Card and J. Mackinlay, "The Structure of the Information Visualization Design Space," Information Visualization, pp. 92-99, 1997.

[4] E. Segel and J. Heer, "Narrative Visualization: Telling Stories with Data," IEEE Transactions on Visualization and Computer Graphics, vol. 16, no. 6, pp. 1139-1148, 2010.

[5] H. Schulz, T. Nocke, M. Heitzler, and H. Schumann, "A Design Space of Visualization Tasks," IEEE Transactions on Visualization and Computer Graphics, vol. 19, no. 12, pp. 2366-2375, 2013.

[6] B. Shneiderman, "The Eyes Have It: A Task by Data Type Taxonomy for Information Visualizations," IEEE Symposium on Visual Languages, pp. 336-343, 1996.

[7] J. Bertin, Semiology of Graphics. Diagrams, Networks, Maps, ESRI Press, 2011.

[8] J. Talbot, V. Setlur, and A. Anand, "Four Experiments on the Perception of Bar Charts," IEEE Transactions on Visualization and Computer Graphics, vol. 20, no. 12, pp. 2152-2160, 2014.

[9] C. Ware, Information Visualization. Perception for Design, Morgan Kaufmann, 2004.

[10] M. Harrower and C. A. Brewer, "ColorBrewer.org: An Online Tool for Selecting Colour Schemes for Maps," The Cartographic Journal, vol. 40, no. 1, pp. 27-37, 2003.

[11] C. Gramazio, K. Schloss, and D. Laidlaw, "The Relation Between Visualization Size, Grouping, and User Performance," IEEE Transactions on Visualization and Computer Graphics, vol. 20, no. 12, pp. 1953-1962, 2014.

[12] T. Munzner, Visualization Analysis and Design. CRC Press, Sept. 2015.

[13] J. Thomas and K. Cook, "Illuminating the Path: The Research and Development Agenda for Visual Analytics," tech. rep., 2005.

[14] W. S. Cleveland and R. McGill, "Graphical Perception: Theory, Experimentation, and Application to the Development of Graphical Methods," Journal of the American Statistical Association, pp. 531-554, 1984.
[15] J. Mackinlay, "Automating the Design of Graphical Presentations of Relational Information," $A C M$ Transactions on Graphics, vol. 5, no. 2, pp. 110-141, 1986.

[16] E. H. Chi, "A taxonomy of visualization techniques using the data state reference model," Information Visualization, 2000. InfoVis 2000. IEEE Symposium on, pp. 69-75, 2000.

[17] D. Keim, G. Andrienko, J. Fekete, C. Görg, J. Kohlhammer, and G. Melançon, "Visual Analytics: Definition, Process, and Challenges," Information Visualization, pp. 154-175, 2008.

[18] B. Lee, C. Plaisant, C. Parr, J. Fekete, and N. Henry, "Task taxonomy for graph visualization," in Proceedings of the 2006 AVI workshop on BEyond time and errors: novel evaluation methods for information visualization, (New York), pp. 1-5, ACM, 2006.

[19] J. Heer and B. Shneiderman, "Interactive Dynamics for Visual Analysis," Queue, vol. 10, no. 2, 2012.

[20] D. Gotz and M. Zhou, "Characterizing Users' Visual Analytic Activity for Insight Provenance," Information Visualization, vol. 8, no. 1, pp. 42-55, 2009.

[21] J. Thomas and K. Cook, "A Visual Analytics Agenda," IEEE Computer Graphics and Applications, vol. 26, pp. 10-13, Jan. 2006.

[22] W. Ribarsky, B. Fisher, and W. Pottenger, "Science of Analytical Reasoning," Information Visualization, vol. 8, no. 4, pp. 254-262, 2009.

[23] X. Wang, D. Jeong, R. Chang, and W. Ribarsky, "RiskVA: A Visual Analytics System for Consumer Credit Risk Analysis," Tsinghua Science and Technology, vol. 17, no. 4, pp. 440-451, 2012.

[24] S. F. Roth, P. Lucas, J. A. Senn, C. C. Gomberg, M. B. Burks, P. J. Stroffolino, A. J. Kolojechick, and C. Dunmire, "Visage: A User Interface Environment for Exploring Information," Wiley Interdisciplinary Reviews: Computational Statistics, 1996.

[25] Y. Kang and J. Stasko, "Characterizing the Intelligence Analysis Process," IEEE Conference on Visual Analytics Science and Technology, pp. 21-30, 2011.

[26] Y. B. Shrinivasan, D. Gotz, and J. Lu, "Connecting the Dots in Visual Analysis," IEEE Symposium on Visual Analytics Science and Technology, pp. 123-130, 2009.

[27] T. M. Green and R. Maciejewski, "A Role for Reasoning in Visual Analytics," in Hawaii International Conference on System Sciences, pp. 1495-1504, 2013.

[28] J. Stasko, C. Görg, and Z. Liu, "Jigsaw: Supporting Investigative Analysis Through Interactive Visualization," Information Visualization, vol. 7, no. 2, 2008.

[29] M. Hossain and C. Andrews, "Helping Intelligence Analysts Make Connections.," Workshops at the Twenty-Fifth AAAI Conference on Artificial Intelligence, pp. 22-31, 2011.

[30] J. Perry, C. D. Janneck, C. Umoja, and W. M. Pottenger, "Supporting Cognitive Models of Sensemaking in Analytics Systems," 2009.

[31] A. Endert, P. Fiaux, and C. North, "Unifying the sensemaking loop with semantic interaction," ... on Interactive Visual Text Analytics for ..., 2011. 
[32] K. Cook, N. Cramer, D. Israel, M. Wolverton, J. Bruce, R. Burtner, and A. Endert, "Mixed-Initiative Visual Analytics Using Task-Driven Recommendations," in IEEE Conference on Visual Analytics Science and Technology (VAST), pp. 9-16, IEEE, 2015.

[33] M. Vartak, S. Huang, T. Siddiqui, and S. Madden, "Towards Visualization Recommendation Systems," IEEE Transactions on Visualization and Computer Graphics, Aug. 2015.

[34] D. Gotz, Z. When, J. Lu, P. Kissa, N. Cao, W. H. Qian, S. X. Liu, and M. Zhou, "HARVEST: An Intelligent Visual Analytic Tool for the Masses," in Proceedings of the First international Workshop on Intelligent Visual Interfaces for Text Analysis, ACM Press, 2010.

[35] D. Gotz and M. Zhou, "An Empirical Study of User Interaction Behavior During Visual Analysis," Tech. Rep. RC24525, 2008

[36] M. Brehmer and T. Munzner, "A Multi-Level Typology of Abstract Visualization Tasks," IEEE Transactions on Visualization and Computer Graphics, vol. 19, no. 12, pp. 2376-2385, 2013.

[37] S. Casner, "Task-Analytic Approach to the Automated Design of Graphic Presentations," ACM Transactions on Graphics, vol. 10, pp. 111-151, Apr. 1991.

[38] M. Zhou and S. Feiner, "Visual Task Charaterisation for Automated Visual Discourse Synthesis," in SIGCHI Conference on Human Factors in Computing Systems, (New York), pp. 392-399, ACM Press, 1998.

[39] R. M. Thiago, L. G. Azevedo, and V. T. da Silva, "CATS: Cognitive Analytic Trail System," in Thirtieth Conference on Artificial Intelligence Symbiotic Cognitive Systems, pp. 768-771, Workshops at the .., 2016.

[40] R. Eccles, T. Kapler, R. Harper, and W. Wright, "Stories in GeoTime," in Proceedings of the IEEE Symposium on Visual Analytics Science and Technology (VAST), pp. 3-17, IEEE Computer Society, 2007.

[41] C. Chen, "Information Visualization," Wiley Interdisciplinary Reviews: Computational Statistics, vol. 2, pp. 387-403, Apr. 2010.

[42] E. R. Tufte, Envisioning Information. Graphics Press, May 1990

[43] E. R. Tufte, Beautiful evidence. Cheshire, Connecticut: Graphics Press LLC, 2006.

[44] S. Few, Show Me the Numbers: Designing Tables and Graphs to Enlighten. Analytics Press, 2nd ed., 2012.

[45] S. Few, Now You See It: Simple Visualization Techniques for Quantitative Analysis. Analytics Press, 1st ed., 2009.

[46] D. McCandless, Knowledge Is Beautiful. Harper Collins, Nov. 2014

[47] D. McCandless, Information is Beautiful. Harper Collins UK, 2009.

[48] R. Kosara and J. Mackinlay, "Storytelling: The Next Step for Visualization," Computer, vol. 46, no. 5, pp. 44-50, 2013.

[49] Y. Tanahashi, C.-H. Hsueh, and K.-L. Ma, "An Efficient Framework for Generating Storyline Visualizations from Streaming Data," IEEE Transactions on Visualization and Computer Graphics, vol. 21, pp. 730-742, June 2015.
[50] J. Hullman and N. Diakopoulos, "Visualization Rhetoric: Framing Effects in Narrative Visualization," IEEE Transactions on Visualization and Computer Graphics, vol. 17, no. 12, pp. 2231-2240, 2011.

[51] E. K. Choe, "Visual Framing: Nudging Toward Health Behavior Change," 2012.

[52] A. V. Pandey, A. Manivannan, O. Nov, M. Satterthwaite, and E. Bertini, "The Persuasive Power of Data Visualization," IEEE Transactions on Visualization and Computer Graphics, vol. 20, pp. 2211-2220, Dec. 2014.

[53] B. Koszegi and M. Rabin, "A Model of Reference-Dependent Preferences," Quarterly Journal of Economics, vol. 121, no. 4, pp. 1133-1165, 2006.

[54] R. Thaler, "Toward a Positive Theory of Consumer Choice," Journal of Economic Behavior \& Organization, vol. 1, no. 1, pp. 39-60, 1980.

[55] D. Kahneman, J. L. Knetsch, and R. Thaler, "Experimental Tests of the Endowment Effect and the Coase Theorem," Journal of Political Economy, 1990.

[56] H. Simon, Models of Bounded Rationality: Empirically Grounded Economic Reason. MIT Press, 1982.

[57] D. Kahneman, "Maps of Bounded Rationality: Psychology for Behavioral Economics," The American Economic Review, vol. 93, no. 5, pp. 1449-1475, 2003.

[58] A. Savikhin, R. Maciejewski, and D. S. Ebert, "Applied Visual Analytics for Economic Decision-Making," IEEE Symposium on Visual Analytics Science and Technology, pp. 107-114, 2008.

[59] I. Levin, S. Schneider, and G. Gaeth, "All Frames are not Created Equal: A Typology and Critical Analysis of Framing Effects," Organizational Behavior and Human Decision Processes, vol. 76, no. 2, pp. 149-188, 1998.

[60] E. K. Choe, J. Jung, B. Lee, and K. Fisher, "Nudging People Away From Privacy-Invasive Mobile Apps Through Visual Framing," in Proceedings of the 33rd Annual ACM Conference on Human Factors in Computing Systems, (Heidelberg), Springer Berlin, IFIP Conference on Human-Computer Interaction, 2013.

[61] A. Tversky and D. Kahneman, "Rational Choice and the Framing of Decisions," Journal of Business, vol. 59, no. 4, pp. S251-S278, 1986.

[62] R. Garcia-Retamero and M. Galesic, "How to Reduce the Effect of Framing on Messages About Health," Journal of General Internal Medicine, vol. 25, pp. 1323-1329, Aug. 2010. 